\title{
Presas y sitios de capturas utilizados por arañas cangrejo (Araneae: Thomisidae y Philodromidae) en un pastizal del centro de Argentina
}

\author{
Hugo Javier Marrero ${ }^{1, \otimes}$; Gabriel Pompozzi ${ }^{2} \&$ Juan Pablo Torretta ${ }^{3}$ \\ 1. Instituto Argentino de Investigaciones de las Zonas Áridas, Conicet, Mendoza, Argentina. 2. Cátedra de Zoología de \\ Invertebrados II, Departamento de Biología, Bioquímica y Farmacia, Universidad Nacional del Sur, Conicet, Bahía Blanca, \\ Argentina. 3. Cátedra de Botánica General, Facultad de Agronomía, Universidad de Buenos Aires, Conicet, Buenos Aires, \\ Argentina
}

\begin{abstract}
Resumen. La distribución de los depredadores y flores son factores importantes que influyen sobre la elección del sitio de forrajeo por los visitantes florales. Los depredadores como las arañas cangrejo pueden determinar la estructura de las comunidades de visitantes florales y afectar la polinización de las plantas. Los objetivos de este trabajo fueron (a) evaluar el uso por las arañas cangrejo de flores o inflorescencias (en adelante unidades de atracción floral, UAF) como sitios de captura; (b) determinar características que pueden afectar el uso de los sitios de captura; y (c) evaluar la relación entre el tamaño de las arañas y el de sus presas. Recorrimos dos parcelas de una hectárea en el centro de la región Pampeana (Buenos Aires, Argentina) registrando todas las plantas entomófilas en flor y sus visitantes florales. Además, capturamos todas las arañas cangrejo con y sin presa registrando las UAF que utilizaron como sitio de captura. Medimos y evaluamos la relación entre el tamaño de las arañas y de sus presas. Las arañas cangrejo utilizaron como sitios de captura las UAF más abundantes y con mayor riqueza de visitantes florales, independientemente de si eran flores o inflorescencias. Por otro lado, el tamaño de la araña podría determinar el tamaño de su presa. Encontramos una asociación positiva entre ambas variables, aunque este resultado fue variable dependiendo de los órdenes taxonómicos de las presas. Estos resultados sugieren que las arañas cangrejo pueden tener un papel relevante modelando la estructura de las interacciones planta-polinizadores.
\end{abstract}

[Palabras clave: polinizador, triple interacción, visitante floral, región Pampeana]

\begin{abstract}
Preys and capture sites used by crab spiders (Araneae: Thomisidae and Philodromidae) in a grassland of central Argentina. Distribution of predators and flowers influence on the selection of forage site of flower visitors. Predators, as crab spiders, can determine the structure of communities of flower visitors and influence on plant pollination. The objectives of this study were: (a) to evaluate the use of flowers or inflorescences (hereinafter units floral attraction, UFA) by crab spiders as capture sites; (B) to identify traits that may affect the use of capture sites; and (c) to assess the relationship between the size of the spiders and their prey. We surveyed two plots of one hectare in center of Pampas region (Buenos Aires, Argentina) recording all entomophilous flowering plants and their floral visitors. In addition, we captured all crab spiders with and without preys, recording the UFA they used as capture site. We estimate and evaluate the relationship between the size of the spiders and their prey. Crab spiders used as capture sites the most abundant UFA and with higher richness of floral visitors, regardless of whether they were flowers or inflorescences. On the other hand, the size of the spider could be determining the size of the preys. We found a positive association between the two variables, although this result was variable depending on the taxonomic orders of preys. Those results suggest that crab spiders can have a relevant role as modelers of the structure of plant - pollinator interaction.
\end{abstract}

[Keywords: Pollinator, triple interaction, floral visitor, Pampas region]

\section{INTRODUCCIÓN}

La distribución de los depredadores y de los recursos florales son dos factores que afectan la elección del sitio de forrajeo por los visitantes florales (Llandres et al. 2012). Los depredadores de visitantes florales, al depredar directamente y/o ahuyentar potenciales polinizadores, pueden reducir la adecuación de las plantas (Dukas 2005; Gonçalves-Souza et al. 2008; Brechbühl et al. 2010a; Tan et al. 2013). Las arañas cangrejo (Araneae: Thomisidae y Philodromidae) son los depredadores de visitantes florales más frecuentes, las cuales capturan sus presas mientras éstas forrajean sobre las flores (Morse 1984; Dukas 2007). Este sistema tripartito (flor-visitante floral-araña cangrejo) combina interacciones mutualistas (polinización) y antagonistas (depredación) conformando una compleja red de interacciones (Marrero et al. 2013). Estas relaciones mutualistas y

Editor asociado: Alejandro Farji-Brener 
antagonistas pueden afectar la estructura de las comunidades (Sauve et al. 2013) y la importancia relativa de las interacciones involucradas (Tadey et al. 2013).

La selección del sitio de captura es importante para las arañas cangrejo debido a que invierten considerables cantidades de energía y tiempo para lograr la captura de sus presas (Morse 2006). Estas arañas (principalmente las pertenecientes a la familia Thomisidae) son conocidas por la posibilidad de cambiar su coloración y por su comportamiento de sentarse y esperar a las presas (Chittka 2001; Bhaskara et al. 2009). Aunque las arañas pueden encontrarse en diferentes partes de la planta, utilizan preferencialmente las flores como sitios de captura (Morse 2007). El motivo por el cual una araña cangrejo utiliza un sitio de captura (flor y/o inflorescencia) puede ser variable y estar relacionado con características comportamentales y/o evolutivas de las especies (Bhaskara et al. 2009; Brechbühl et al. 2010b). Las arañas cangrejo utilizan señales visuales, olfativas y/o táctiles para encontrar sus sitios de captura (Heiling et al. 2004). Sin embargo, estas características son variables; por ej., algunas arañas escogen como sitios de captura flores que emiten aromas (Heiling et al. 2004) y capaces de reflejar la luz ultravioleta (UV) (Bhaskara et al. 2009; Defrize et al. 2010). Otras especies de arañas cangrejo son capaces de reflejar el UV y seleccionan flores sin reflectancia de UV para aumentar el contraste y así atraer a los visitantes florales (Heiling et al. 2003; Heiling et al. 2005; Bhaskara et al. 2009; Llandres et al. 2011). Asimismo, la elección del sitio de captura podría estar influenciado por otros factores como las experiencias de capturas (aprendizaje) y/o la disponibilidad de recursos florales (por ej. estado fenológico de las plantas de la comunidad) (Morse 2000). Adicionalmente, la elección por parte de las arañas cangrejo también puede estar determinada por la característica de las plantas con flor. Por ejemplo, esperar visitantes florales en plantas generalistas (es decir, plantas con flores visitadas por una gran riqueza de visitantes florales), aumentaría la probabilidad de capturar una mayor diversidad de presas y obtener una dieta más variada, lo que le podría dar una ventaja nutricional a la araña. Por otro lado, las arañas cangrejo podrían usar preferencialmente inflorescencias en comparación con flores individuales, debido a que en las inflorescencias podría haber más recursos disponibles para los visitantes florales, y estar disponible por tiempo más prolongado. Esto podría volver más atractivas las inflorescencias para las presas potenciales de las arañas, convirtiendo a las inflorescencias en los sitios de capturas seleccionados por las arañas cangrejo.

Las arañas cangrejo atacan diversos visitantes florales, sin embargo, muestran preferencia por presas más vulnerables, como aquellas de menor tamaño, sin aguijón, sin defensas químicas y con cutículas más delicadas (Cheli et al. 2006, Marrero et al. 2013). Varios estudios han sugerido que la identidad de un visitante floral podría desempeñar un papel importante en las respuestas de captura que muestran las arañas (Brechbühl et al. 2010a, y citas allí). Por otra parte, el tamaño de los visitantes florales es un factor que podría determinar la selección de las presas por las arañas cangrejo (Morse 2000; Dukas \& Morse 2003, 2005; Brechbühl et al. 2010a). Brechbühl et al. (2010a) mencionan que algunas de las especies de visitantes florales de pequeño tamaño en su estudio, evitaron alimentarse en flores con presencia de arañas, mientras que las de mayor tamaño no modificaron su comportamiento. En este caso, las arañas podrían no seleccionar su presa y sólo capturar los visitantes florales disponibles, que no son capaces de evitarlas. Por otra parte, el éxito de la captura podría estar relacionado con la frecuencia de encuentros (Cheli et al. 2006; Brechbühl et al. 2011), por lo que las presas más frecuentes podrían ser los visitantes florales más abundantes de la comunidad. Una mayor frecuencia de encuentros, podría generar una mayor experiencia en la captura de una presa en particular (Brechbühl et al. 2011). Entender cuáles son los parámetros que las arañas cangrejo utilizan para la elección de sus presas ayudaría a comprender mejor la elección de sus sitios de captura (y viceversa).

El objetivo de este trabajo fue investigar el uso de sitios de captura y las presas consumidas por las arañas cangrejo en una comunidad de pastizal en la Región Pampeana (Argentina). Particularmente, analizamos el tipo de sitios de captura más frecuentemente utilizado por las arañas (flor o inflorescencia), y la abundancia de cada uno de estos tipos entre las plantas entomófilas florecidas en el área de estudio. Por otro lado, evaluamos la relación entre el tamaño corporal de las arañas y el de las presas. Y finalmente, discutimos algunos de los mecanismos por los cuales las arañas cangrejo podrían seleccionar a sus sitios de captura y a sus presas. 


\section{Materiales y MÉTODOS}

\section{Sistema de estudio}

El trabajo se realizó en la Estancia San Claudio (Partido de Carlos Casares, Provincia de Buenos Aires, Argentina), en la subregión pampeana denominada Pampa Interior. Su clima es templado subhúmedo, con $1022 \mathrm{~mm}$ de precipitación anual media para los últimos 25 años. Las temperaturas medias mensuales varían de $7.2^{\circ} \mathrm{C}$ en julio a $23.8^{\circ} \mathrm{C}$ en enero (Tognetti et al. 2010). Los sitios de captura y las presas consumidas por las arañas cangrejos se muestrearon en dos parcelas de una hectárea separadas por $6 \mathrm{~km}$ sin manejo agrícolaganadero por más de 20 años (Marrero et al. 2014). Se utilizaron estas dos parcelas debido a que para ambas se tenía conocimiento de la comunidad de plantas entomófilas y visitantes florales por trabajos previos (Marrero et al. 2013; 2014). Las parcelas difieren en la composición de la comunidad de plantas (principalmente gramíneas), pero presentan similar fisonomía (Tognetti et al. 2010) y las plantas entomófilas más abundantes de la comunidad se encuentran presentes en ambas parcelas.

Las arañas cangrejo (Araneae: Thomisidae y Philodromidae) tienen amplia distribución y son reconocidas por capturar visitantes florales mientras éstos se alimentan en las flores (Morse 2007). Estas arañas se caracterizan por tener los dos pares de patas anteriores raptoras grandes y la tendencia a correr hacia los lados (Legrand \& Morse 2000). Asimismo, una característica distintiva de estas arañas (particularmente las pertenecientes a la familia Thomisidae), es su capacidad para cambiar la coloración del tegumento dependiendo del sustrato ocupado. Pueden variar la tonalidad entre blanco, amarillo, purpura y rosa dependiendo de la especie (Chittka 2001; Heiling et al. 2004; Brechbühl et al. 2010b). Esta cualidad les permite mimetizarse en las flores y emboscar a sus presas mientras forrajean por alimento (Morse 2007).

\section{Muestreos}

Realizamos los muestreos entre diciembre 2010 y marzo de 2011. Visitamos las parcelas con frecuencia mensual y en cada oportunidad realizamos un muestreo de cuatro horas (dos horas por la mañana: 10:00-12:00, y dos horas por la tarde: 16:00-18:00) en cada parcela (16 horas de esfuerzo de muestreo total por parcela). En cada muestreo, dos observadores recorrimos lentamente la parcela (media parcela cada observador) de manera exhaustiva y evitando pasar por el mismo lugar.

Plantas entomófilas: Durante el recorrido de la parcela, observamos y registramos la riqueza de plantas entomófilas florecidas y la abundancia de unidades de atracción floral (UAF - flores o inflorescencias) de cada especie. Contabilizamos, con un contador manual, todas las UAF observadas en cada parcela de estudio. Todas las especies fueron colectadas, herborizadas y determinadas en el laboratorio.
Visitantes florales: Durante los recorridos en las parcelas, registramos la riqueza de visitantes florales. Capturamos aquellos visitantes florales que no pudimos identificar y que fueron observados forrajeando en las flores entomófilas. Estos visitantes fueron sacrificados in situ, y en el laboratorio se montaron sobre alfileres entomológicos para su posterior determinación. A cada visitante floral se le registró la especie de planta huésped.

Arañas cangrejo y sus presas: Capturamos las arañas cangrejo que se encontraron sobre las UAF a la espera de un visitante floral, y aquellas que se encontraron consumiendo un visitante floral. Debido a que estas arañas consumen visitantes florales, en este trabajo supusimos que todos los visitantes florales son potenciales presas de estas arañas, y en adelante, cualquier visitante floral consumido es llamado presa potencial. Una vez observada sobre las UAF, capturamos manualmente las arañas cangrejo (con o sin presa potencial) y las colocamos en frascos individuales con alcohol $70 \%$ para su conservación y posterior determinación. En algunas ocasiones, se capturaron más de un espécimen de araña cangrejo sobre la misma UAF. Para las arañas (y sus presas potenciales), registramos la especie de planta huésped. Solo capturamos las arañas cangrejo de tamaño corporal mayor a $2 \mathrm{~mm}$, que incluyeron tanto a individuos adultos (32\% del total) como juveniles (68\%). Las arañas de menor tamaño corporal son más difíciles de encontrar sobre las flores que las de mayor tamaño, por lo cual estaremos subestimando la abundancia de estas arañas.

Estimación de los tamaños corporales: En el laboratorio y bajo microscópico binocular (60X de aumento) medimos las arañas cangrejo y sus presas potenciales mediante una lente grillada. Las medidas tomadas en las especies presa potencial fueron: ancho del tórax y largo total del cuerpo (desde el extremo de la cabeza hasta el extremo del abdomen); mientras que para las arañas se midió ancho y largo del cefalotórax (Arnqvist \& Henriksson 1997). Con estos datos obtuvimos medidas morfométricas del área de los cuerpos de las presas potenciales y arañas estimadas como el área de un elipse $=\pi \times($ ancho /2) $\times($ largo /2).

\section{Análisis de datos}

Para llevar a cabo los análisis unimos los datos de ambas parcelas. Para evaluar el uso de los sitios de captura realizamos correlaciones utilizando como variables la abundancia de UAF de cada especie de planta huésped para las arañas; la riqueza de visitantes florales (número de especies) que forrajearon sobre estas especies vegetales y la abundancia de arañas cangrejo encontradas en cada especie de planta huésped. Para este análisis sólo utilizamos las especies de plantas que tuvieron interacciones con visitantes florales y con arañas cangrejo, para evitar los sesgos en la correlación asociada a valores nulos. Por otro lado, para determinar el uso más frecuente de flores o 
inflorescencias como sitios de captura, realizamos la prueba U de Mann-Whitney (debido a que los datos no tuvieron una distribución normal), comparando la abundancia de arañas cangrejo entre los dos tipo de UAF. Para evaluar la relación entre los tamaños corporales de las arañas y sus presas potenciales, realizamos una prueba de Kruskal-Wallis de las medidas morfométricas estimadas de las presas potenciales para comparar el tamaño entre las especies de distintos órdenes taxonómicos. Adicionalmente, realizamos correlaciones lineales utilizando como variables, las medidas morfométricas estimadas de las presas potenciales y de las arañas cangrejo. Hicimos una correlación para el total de las presas potenciales (todos los órdenes de visitantes florales que fueron presas potenciales) y para cada orden taxonómico por separado (excepto Orthoptera y Coleoptera que estuvieron representados por un único individuo). Los datos utilizados en las correlaciones fueron ln transformados para cumplir con el supuesto de normalidad. El análisis de los datos se realizó con el programa de estadística Infostat (Di Rienzo et al. 2008).

\section{Resultados}

Entre las dos parcelas capturamos 559 arañas cangrejo pertenecientes a cinco especies (cuatro especies de Thomisidae y una de Philodromidae) (Apéndice 1-a, Información Suplementaria), de las cuales, el $21.5 \%$ se encontraron consumiendo alguna presa potencial. Principalmente, las presas potenciales fueron Diptera $(60 \%$ de los individuos pertenecientes al $56 \%$ de las especies) e Hymenoptera $(32 \%, 25 \%)$ y en menor medida, Lepidoptera $(6 \%, 14 \%)$, Orthoptera $(1 \%, 2.5 \%)$ y Coleoptera $(1 \%$, $2.5 \%$ ) (Figura 1) (Apéndice 1-b, Información Suplementaria). Las arañas fueron encontradas sobre flores y/o inflorescencias de 20 especies de plantas entomófilas. En total observamos

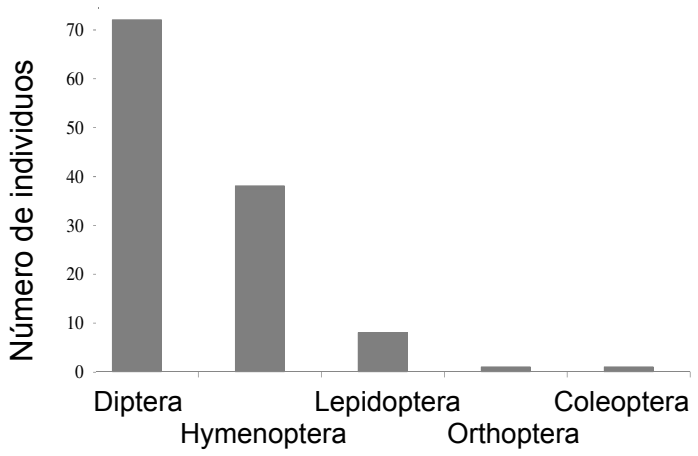

Figura 1. Número de visitantes florales pertenecientes a diferentes órdenes taxonómicos de insectos capturados por las arañas cangrejo.

Figure 1. Number of floral visitors caught by crab spiders according to the taxonomic order of insects.

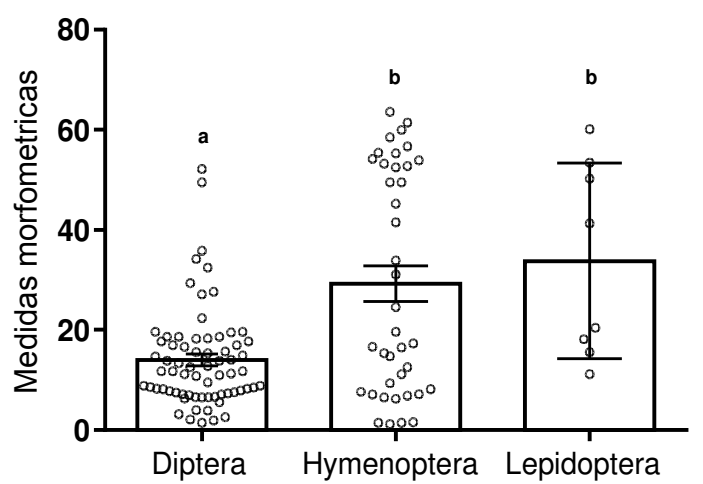

Figura 2. Medidas morfométricas de las presas (media \pm ES) capturadas por las arañas cangrejo. Las medidas fueron calculadas como el área de un elipse $=\pi \mathrm{x}$ (ancho /2) x (largo /2). Cada punto corresponde a una presa perteneciente a los órdenes más abundantes de visitante floral (Diptera, Hymenoptera y Lepidoptera). Letras diferentes sobre las columnas indican diferencias significativas.

Figure 2. Morphometric measurements of preys (mean \pm $\mathrm{SE}$ ) caught by crab spiders. The measures were calculated as an ellipse area $=\pi \times$ (width /2) $\times$ (length /2). Each point corresponds to one prey belonging to the most abundant floral visitor orders (Diptera, Hymenoptera and Lepidoptera). Letters above bar indicate significant

42 especies de plantas entomófilas en flor (Apéndice 1-c), y en 25 de ellas se capturaron 134 especies de visitantes florales (51 Hymenoptera, 47 Diptera, 19 Coleoptera, 14 Lepidoptera, 1 Orthoptera, 1 Hemiptera y 1 Blattaria) (Apéndice 1-b).

Las arañas cangrejo fueron más abundantes en las flores de las plantas entomófilas más abundantes $(\mathrm{r}=0.57, p=0.03)$ y con mayor riqueza de visitantes florales $(\mathrm{r}=0.73, p=$ 0.001). Por otro lado, las plantas con mayor abundancia de UAF tuvieron interacciones con una mayor riqueza de visitantes florales $(\mathrm{r}=$ $0.58, p=0.02$ ). No se encontró que las arañas utilicen más algún tipo de UAF, ya que la abundancia de arañas fue similar sobre flores $\mathrm{o}$ inflorescencias $(\mathrm{N}=20, \mathrm{~W}=28, p=0.18)$.

Las especies presa potencial pertenecientes al orden Diptera fueron más pequeñas que las especies pertenecientes a los órdenes Hymenoptera y Lepidoptera $(\mathrm{N}=118, \mathrm{H}=$ 13.68, $p=0.001$ ) (Figura 2). Las correlaciones entre el tamaño de las arañas cangrejo y sus presas potenciales, resultaron positivas y significativas para el total de las presas potenciales y para Diptera e Hymenoptera y positiva con una tendencia marginalmente significativa para Lepidoptera (Tabla 1, Figura 3). Las arañas más grandes fueron encontradas consumiendo dípteros e himenópteros de mayor tamaño, en comparación a las arañas de menor tamaño. Esta relación entre las 


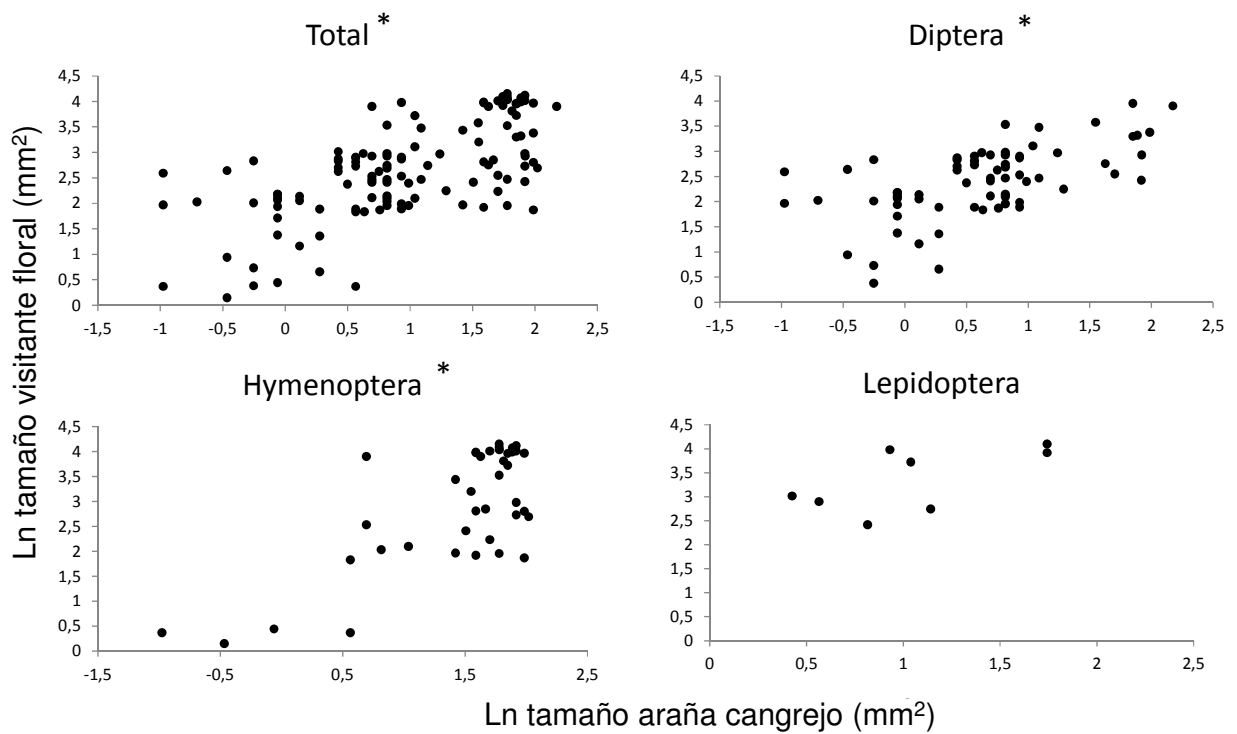

Figura 3. Correlaciones lineales simples entre las medidas morfométricas estimadas de los visitantes florales (ancho de tórax y largo total del cuerpo) y las arañas cangrejo (ancho y largo de tórax). Las medidas fueron transformadas logarítmicamente. Se realizaron cuatro correlaciones, con el total de los visitantes florales (Total) y con cada orden por separado (Diptera, Hymenoptera y Lepidoptera). Las gráficas en donde se muestra el * son las que resultaron significativas $(\mathrm{p}<0.05)$.

Figure 3. Correlation between morphometric measurements of floral visitors (thorax width and total body length) and crab spiders (width and length of thorax). Measurements were ln transformed. Four correlation were performed with total floral visitors (Total) and each order separately (Diptera, Hymenoptera and Lepidoptera). The graphics where * are shown were significant $(\mathrm{p}<0.05)$.

variables de tamaño corporal fue explicada mejor para el orden Hymenoptera que para el orden Diptera (Tabla 1).

Tabla 1. Valores de las correlaciones lineales realizadas entre las medidas morfométricas de las arañas cangrejo y sus presas. Se realizaron cuatro correlaciones, con el total de los visitantes florales (Total) y con cada orden por separado (Diptera, Hymenoptera y Lepidoptera). Los valores $p$ en negrita indican relaciones significativas entre variables.

Table 1. Values of the correlation between morphometric measures of crab spiders and their preys. Four correlation were performed with total floral visitors (Total) and each order separately (Diptera, Hymenoptera and Lepidoptera). The p-values in bold indicate significant relationships between variables.

\begin{tabular}{lcccc}
\hline Órdenes & $\mathrm{N}$ & $\mathrm{R}^{2}$ & $\mathrm{~T}$ & Valor- $p$ \\
\hline Total & 116 & 0.43 & 9.36 & $<\mathbf{0 . 0 0 0 1}$ \\
Diptera & 69 & 0.36 & 6.19 & $<\mathbf{0 . 0 0 0 1}$ \\
Hymenoptera & 39 & 0.54 & 6.53 & $<\mathbf{0 . 0 0 0 1}$ \\
Lepidoptera & 8 & 0.41 & 2.04 & 0.087 \\
\hline
\end{tabular}

\section{DisCUSIÓN}

Los sitios de captura más comúnmente utilizados por las arañas cangrejo fueron las plantas de la comunidad con mayor abundancia de UAF (independientemente del tipo de UAF) y que recibieron visitas más diversas. Asimismo, las arañas de mayor tamaño capturaron presas potenciales más grandes que las de menor tamaño, aunque este resultado varió dependiendo del orden de los visitantes florales consumidos por las arañas.

La abundancia de arañas en las plantas estuvo asociada a una mayor abundancia de UAF y riqueza de visitantes florales. Nuestros resultados concuerdan con los reportados por Hanna \& Eason (2013) (ver también Morse 2000 y citas allí), quienes encontraron que juveniles de la araña cangrejo Mecaphesa asperata (Thomisidae) no utilizaron la abundancia de presas para evaluar los sitios de forrajeo, sino que prefirieron sitios de captura que atrajeron una mayor riqueza de presas y eligieron las inflorescencias con mayor número de flores en antesis, sobre inflorescencias con mayor número de flores senescentes o en pimpollos. Sin embargo, en ese trabajo Hanna \& Eason (2013) no evaluaron el uso de los sitios de captura en relación a su abundancia. Es posible que las plantas con mayor abundancia de UAF generen parches más visibles y atraigan con mayor frecuencia a visitantes florales y arañas. Chien \& Morse (1998) sugieren que la presencia de presas es el factor más importante en la selección del sitio de captura por parte de la araña. También comentan que puede influir el estado de la inflorescencia (cantidad de flores en antesis), 
o incluso existir un efecto aditivo entre ambas variables. Juveniles recientemente emergidos de Misumena vatia (Thomisidae) seleccionan inflorescencias de Solidago canadensis (Asteraceae) con baja densidad de flores, pero en pocas horas se desplazan a inflorescencias con mayor cantidad de flores abiertas para disminuir los riesgos de depredación (Morse 2006). Sin embargo, la cantidad de flores en antesis y la diversidad de presas podrían no ser independientes. Análogo a nuestros resultados, una mayor cantidad de flores en antesis, genera una mayor cantidad de recurso y podrían atraer una mayor diversidad de visitantes florales.

No encontramos que las arañas cangrejo utilizaran diferencialmente como sitios de captura flores o inflorescencias. Es posible que el tamaño del "parche" floral sea más relevante que el tipo de UAF. El tamaño, el color y el aroma de las flores/inflorescencias podrían ser características que las arañas cangrejo consideren para evaluar los sitios de captura (Morse 2000), aunque también podría haber decisiones intrínsecas de cada una de las especies estudiadas. Heiling et al. (2004) reportan que el aroma, y no el tamaño de las inflorescencias, indicó una preferencia del sitio de captura por la araña cangrejo Thomisus spectabilis (Thomisidae) sobre inflorescencias de Chrysanthemum frutescens (Astereceae). No obstante, mayor cantidad de flores abiertas, podría emitir más aroma. Por otro lado, el aroma parecería ser la señal floral que utilizan las abejas para identificar las flores de mayor calidad, y las arañas cangrejo explotarían esta característica para encontrar a las abejas (Heiling et al. 2004).

Las arañas de mayor tamaño parecen ser capaces de capturar visitantes florales más grandes. Este hecho podría deberse a que poseen mayor cantidad de veneno que una araña cangrejo de menor tamaño (Foelix 2011) o a que tienen más fuerza y pueden capturar y retener presas más grandes. Las presas potenciales más pequeñas fueron Diptera. En un trabajo previo, se obervó que estas presas son las más comunes, aún sin ser los visitantes florales más frecuentes, posiblemente debido a su falta de defensa y a su cutícula blanda (Marrero et al. 2013). Los himenópteros son menos comunes que los dípteros como presas de las arañas cangrejo, pero tienen mayor tamaño corporal, y en el caso de las hembras poseen aguijones con el que podrían defenderse. No obstante, el tamaño de las presas no se relaciona necesariamente con su vulnerabilidad (Brechbühl et al. 2011). La vulnerabilidad se relaciona más con la falta de habilidad para reconocer a las arañas y evitarlas (Brechbühl et al. 2011). Algunas abejas podrían aprender a reconocer y a evitar a las arañas cangrejo, mientras que las moscas podrían no reconocerlas (Brechbühl et al. 2010a). Más aún, abejas solitarias de pequeño tamaño son las que aprenden a evitar las arañas, mientras que las abejas sociales de mayor tamaño no lo hacen (Brechbühl et al. 2010a). Según Brechbühl et al. (2011), el tamaño y la identidad de la presa son los factores que determinan el éxito en la captura por las arañas cangrejo. Además, para capturar presas de gran tamaño las arañas deben invertir más tiempo y energía, y este desbalance podría verse beneficiado con una mayor captura de presas de menor tamaño (Uetz 1992; Brechbühl et al. 2011).

La riqueza de visitantes florales podría determinar la elección del sitio de captura por las arañas cangrejo. Como fue explicado anteriormente, sitios con una diversidad mayor de visitantes florales podrían ofrecer una mayor diversidad de dieta a las arañas, e incrementar la probabilidad de encontrar en dicha diversidad de visitas individuos más vulnerables a la depredación. También es posible que las arañas de mayor tamaño puedan seleccionar una mayor diversidad de presas potenciales y competir por mejores sitios de captura que las arañas de menor tamaño.

En resumen, encontramos que las arañas cangrejo eligen los sitios de captura con mayor abundancia de estructuras florales, independientemente de si éstas eran flores o inflorescencias, y con mayor riqueza de visitantes florales. Adicionalmente, encontramos que el tamaño de las presas potenciales depende positivamente del tamaño de la araña. Futuros trabajos deberían analizar el efecto en la adecuación de las arañas de dietas que varíen en la riqueza de presas, y realizar observaciones y experimentos para determinar los mecanismos concretos por los cuales el tamaño de la presa puede ser un limitante en el proceso de captura y depredación por parte de la araña. Adicionalmente, se debería estudiar, por separado, las diferentes especies de arañas cangrejo, e incluir en los análisis los diferentes estados fenológicos de las arañas. Creemos que este trabajo aporta información importante para este tipo de interacciones tripartitas, las cuales han sido poco investigados en el país, y esperamos que 
este estudio funcione como línea de base para generar nuevo conocimiento en el área de la biología de las arañas cangrejo.

Agradecimientos: A la Universidad de Buenos Aires, por el apoyo logístico y el permiso para trabajar en la Estancia San Claudio. A AG Farji-Brener y a dos revisores anónimos por las valiosas sugerencias. El trabajo de campo fue financiado por ANPCyT (PICT 0851). HJM, GP y JPT son miembros de CONICET.

\section{BiBLIOGRAFíA}

ARnQvist, G \& S HenRIKSSON. 1997. Sexual cannibalism in the fishing spider and a model for the evolution of sexual cannibalism based on genetic constraints. Evol Ecol, 11:255-273.

Bhaskara, MR; CM BRIJESH; S AHMEd \& RM Borges. 2009. Perception of ultraviolet light by crab spiders and its role in selection of hunting sites. J Comp Physiol, 195:409-417.

BRECHBÜHL, R; C KROPF \& S BACHER. 2010a. Impact of flowerdwelling crab spiders on plant-pollinator mutualisms. Basic Appl Ecol, 11:76-82.

BRECHBÜHL, R; J CASAS \& S BACHER. 2010b. Ineffective crypsis in a crab spider: a prey community perspective. $P R$ Soc $B, 277: 739-746$

BRECHBÜHL, R; J CASAS \& S BACHER. 2011. Diet choice of a predator in the wild: overabundance of prey and missed opportunities along the prey capture sequence. Ecosphere, 2:133.

Cheli, G; A Armendano \& A GonzÁlez. 2006. Preferencia alimentaria de arañas Misumenops pallidus (Araneae: Thomisidae) sobre potenciales insectos presa de cultivos de alfalfa. Rev Biol Trop, 54: 505-513.

CHIEn, SA \& DH Morse. 1998. The role of prey and flower quality in the choice of hunting sites by adult male crab spiders Misumena vatia (Araneae, Thomisidae). J Arachnol, 26:238-243.

ChitTKA, L. 2001. Camouflage of predatory crab spiders on flowers and the colour perception of bees (Aranida: Thomisidae/Hymenoptera: Apidae). Entomol Gen, 25 (3): 181-187.

Defrize, J; M THÉRY \& J CASAS. 2010. Background colour matching by a crab spider in the field: a community sensory ecology perspective. J Exp Biol, 213: 1425-1435.

Di Rienzo, JA; F CASANOVES; MG Balzarini; L Gonzalez; M TABladA \& CW Robledo. 2008. InfoStat, versión 2008, Grupo InfoStat, FCA, Universidad Nacional de Córdoba, Córdoba.

DukAs, R \& DH MORSE. 2003. Crab spiders affect flower visitation by bees. Oikos, 101 :157-163.

DUKAS, R \& DH MORSE. 2005. Crab spiders show mixed effects on flower-visiting bees and no effect on plant fitness components. EcoScience, 12:244-247.

DuKAS, R. 2005. Bumble bee predators reduce pollinator density and plant fitness. Ecology, 86:1401-1406.

Foelix, R. 2011. Biology of spiders. Third edition. Oxford, RU: Oxford University Press. 419 pp.

Gonçalves-Souza, T; PM OmenA; JC SOuZA \& GQ Romero. 2008. Trait-mediated effects on flowers: artificial spiders deceive pollinators and decrease plant fitness. Ecology, 89:2407-2413.

GonzÁlez, A; G Liljesthröm; E Minervino; D Castro;
S González \& A Armendano. 2009. Predation by Misumenops pallidus (Araneae: Thomisidae) on insect pests of soybean cultures of Buenos Aires Province, Argentina. J Arachnol, 37:282-286.

Hanna, CJ \& PK EAson. 2013. Juvenile crab spiders (Mecaphesa asperata) use indirect cues to choose foraging sites. Ethol Ecol Evol, 25:161-173.

Heiling, AM; ME Herberstein \& L Chittka. 2003. Crabspiders manipulate flower signals. Nature, 421: 334.

Heiling, AM; K Cheng \& ME Herberstein. 2004. Exploitation of floral signals by crab spiders (Thomisus spectabilis, Thomisidae). Behav Ecol, 15:321-326.

Heiling, AM; K Cheng; L Chittka; A Goeth \& ME HERBERSTEIN. 2005. The role of UV in crab spider signals: effects on perception by prey and predators. J Exp Biol, 208:3925-3931.

LEGRAND, RS \& DH MORSE. 2000. Factors driving extreme sexual size dimorphism of a sit-and-wait predator under low density. Biol J Linn Soc, 71:643-664.

Llandres, AL; FM GawryszewsKi; AM HeIling \& ME HerbersteIN. 2011. The effect of colour variation in predators on the behaviour of pollinators: Australian crab spiders and native bees. Ecol Entomol, 36:72-81.

Llandres, AL; E De MAs \& MA Rodríguez-Gironés. 2012. Response of pollinators to the trade-off between resource acquisition and predator avoidance. Oikos 121: 687-696.

Marrero, HJ; JP Torretta \& G Pompozzi. 2013. Triple interaction network among flowers, flower visitors and crab spiders in a grassland ecosystem. Stud Neotrop Fauna E, 48:153-164.

Marrero, HJ; JP Torretta \& D Medan. 2014. Effect of land use intensification on specialization in plant-floral visitor interaction networks in the Pampas of Argentina. Agr Ecosyst Environ, 188:63-71.

Morse, DH. 1984. How crab spiders (Araneae: Thomisidae) hunt at flowers. J Arachnol, 12, 307-316.

Morse, DH. 2000. Flower choice by naïve young crab spiders and the effect of subsequent experience. Anim Behav, 59:943-951.

Morse, DH. 2006. Fine-scale substrate use by a small sitand-wait predator. Behav Ecol, 17:405-409.

Morse, DH. 2007. Predator upon a flower: life history and fitness in a crab spider. Cambridge, MA: Harvard University Press. 377 pp.

Sauve, AMC; C Fontaine \& E ThéBault. 2013. Structurestability relationships in networks combining mutualistic and antagonistic interactions. Oikos, 123: 378-384.

TADEY, M; R AYAZO; F CARRASCO-Rueda; Y CHRISTOPHER; M Domínguez; G La Quay-Velázquez \& M San José. 2013. Depredación de arañas hacia visitantes florales y herbívoros, balance entre mutualismo y antagonismo. Ecología Austral 23:126-134.

TAN, K; Z Hu; W CHEN; Z WANG; Y WANG \& JC NIEH. 2013. Fearful foragers: honey bees tune colony and individual foraging to multi-predator presence and food quality. PLOS ONE 8: e75841.

Tognetti, PM; EJ Chaneton; M OMacini; HJ Trebino \& RJC LEÓN. 2010. Exotic vs. native plant dominance over 20 years of old-field succession on set-aside farmland in Argentina. Biol Conserv, 143: 2494-2503.

Uetz, G. 1992. Foraging strategies of spiders. Trends Ecol Evol, 7:155-159. 